\title{
A 1.5 GeV Compact Light Source with Superconducting Bending Magnets
}

\author{
A.A. Garren, a,b,c D.B. Cline, a,b M.A. Green, ${ }^{c}$ D.E. Johnson, ${ }^{a}$ J.J. Kolonko, a,b \\ E.M. Leung, d D.D. Madura, ${ }^{d}$ and L.C. Schachinger ${ }^{a}$ \\ aParticle Beam Lasers, Inc., Northridge, CA 91324 \\ bUCLA Center for Advanced Accelerators, Los Angeles, CA 90095 \\ cAccelerator and Fusion Research Division, Lawrence Berkeley Laboratory, \\ University of California, Berkeley, CA 94720 \\ dMartin Marietta Technologies, Inc., Rancho Bernardo, CA 92125
}

May 1995

\section{DISCLAIMER}

\begin{abstract}
This report was prepared as an account of work sponsored by an agency of the United States Government. Neither the United States Government nor any agency theregf, nor any of their employees, makes any warranty, express or implied, or assumes any legal liability or responsibility for the accuracy, completeness, or usefulness of any information, apparatus, product, or process disclosed, or represents that its use would not infringe privately owned rights. Reference herein to any specific commercial product, process, or service by trade name, trademark, manufacturer, or otherwise does not necessarily constitute or imply its endorsement, recommendation, or favoring by the United States Government or any agency thereof. The views and opinions of authors expressed herein do not necessarily state or reflect those of the United States Government or any agency thereof.
\end{abstract}

This work was supported by the Office of High Energy and Nuclear Physics, of the U.S. Department of Energy under Contract No. DE-FG03-94ER81826, and Contract No. DE-AC03-76SF00098. 


\section{DISCLAIMER}

Portions of this document may be illegible in electronic image products. Images are produced from the best available original document. 


\title{
A $1.5 \mathrm{GeV}$ Compact Light Source with Superconducting Bending Magnets ${ }^{*}$
}

\author{
A A. Garren a,b,c, D. B. Cline ${ }^{a, b}$, M. A. Green ${ }^{c}$, D. E. Johnson ${ }^{a}$, J J. Kolonko ${ }^{a, b}$, \\ E. M. Leungd, D. D. Madurad and L. C. Schachinger ${ }^{a}$ \\ a. Particle Beam Lasers, Inc., Northridge CA 91324 \\ b. UCLA Center for Advanced Accelerators, Los Angeles CA 90095 \\ c. Lawrence Berkeley Laboratory, Berkeley CA 94720 \\ d. Martin Marietta Technologies Inc., Rancho Bernardo CA 92125
}

\begin{abstract}
This paper describes the design of a compact electron synchrotron light source for producing $X$-rays for medical imaging, protein crystallography, nano-machining and other uses up to $35 \mathrm{keV}$. The source will provide synchrotron light from six 6.9 tesla superconducting $60^{\circ}$ bending magnet stations. In addition the ring, contains conventional quadrupoles and sextupoles. The light source has a circumference of 26 meters, which permits it to be located in a variety of industrial and medical facilities.
\end{abstract}

\section{BACKGROUND}

The machine presented here is designed primarily as a light source for industrial and medical applications. Modified versions of the design may have other applications as a damping ring or collider.

The ring is designed to produce $X$ rays with energies up to $35 \mathrm{keV}$. To make the ring compact, its energy is limited to $1.5 \mathrm{GeV}$. This beam energy allows use of conventional quadrupoles and sextupoles without greatly increasing the ring circumference. In order to get useful quantities of $\mathrm{X}$ rays at $35 \mathrm{keV}$ or above, the bending dipole should have a central induction above $6 \mathrm{~T}$. For the machine described, with a bending induction of $6.9 \mathrm{~T}$ and beam energy of $1.5 \mathrm{GeV}$, the critical synchrotron-radiation energy is $10.3 \mathrm{keV}$. The lattice has been designed to optimize dynamic aperture, emittance, cost, and ring size, while providing adequate space for the If and injection systems.

\section{THE COMPACT STORAGE RING LATIICE}

The racetrack-shaped ring has a hybrid magnet structure, consisting of superconducting dipoles and conventional quadrupoles and sextupoles. The two arcs are identical and each is symmetric about its center. The straight sections bave the same length but different structure. Each is symmetric about its center, so the ring is reflectionsymmetric about the line joining the center points. Figure 1 shows the ring layout.

Each arc consists of six $30^{\circ}$ dipoles, four quadrupoles and six sextupoles. The dipoles are grouped into three pairs, each of which shares a single cryostat. Conceptually, the arc is built up from three cells, where the outer two cells are

\footnotetext{
- This work was performed with the support of the Office of High Energy and Nuclear Physics, United States Department of Energy under contract number DE-FG03-94ER81826 and LBL contract number DE-AC03-76SF00098.
}

truncated beyond the two dipoles. Referring to Figure 1, the center cell consists of the magnet sequence (SFH QF SD B B SD QF SFH), the first cell is (B B SD QF SFH) and the last cell is (SFH QF SD B B). Here SFH is a half focusing chromaticity sextupole, $\mathrm{QF}$ a focusing quadrupole, $\mathrm{SD}$ a defocusing chromaticity sextupole, and $B$ is a dipole.

The rectangular dipole has a field of $6.9 \mathrm{~T}$ and zero gradient. Vertical focusing comes from its edges. The cell has horizontal and vertical phase advances of $120^{\circ}$ and $90^{\circ}$ respectively; these values are obtained by adjusting the strength of $\mathrm{QF}$ and the distance between $\mathrm{QF}$ and $\mathrm{B}$. The horizontal phase advance of three cells is $360^{\circ}$, so the arc is an achromat and the dispersion is zero in the straight sections (see Figure 2). The vertical phase advance is chosen to make the arc as short and the emittance as low as possible.

The straight section at the top of Figure 1 has a 1.6 meter drift in the center for injection. The straight section at the bottom of Figure 1 has seven quadrupoles, between which the if modules would be located. Both straight sections are adjusted so that the periodic beta functions in the arc are brought to waists at the straight section centers. Consequently the emittance depends on the arc design only, not on the straight sections or the tunes. Table I presents the basic parameters for the Lattice.

The bottom straight section produces $3 \pi$ horizontal and vertical phase differences between the centers of the two arcs. Thus, each magnet in one arc is paired with one in the other so as to partially cancell their nonlinear effects. The top straight section quadrupoles are adjusted to make the tunes favorable with respect to resonances, minimization of beam size and chromaticity and the provision of useful drift spaces.

Figure 2 shows the lattice and orbit functions of the ring, running clockwise from the center of the top straight section.

The following variations have been investigated:

1) The arc was composed of either three $60^{\circ}$ dipoles or six $30^{\circ}$ dipoles.

2) Values of the cell vertical phase advance of $120^{\circ}, 90^{\circ}$, and $60^{\circ}$ were compared.

3) The number and location of the defocusing chromaticity SD sextupoles have been varied.

4) Different ring tune values were compared. 


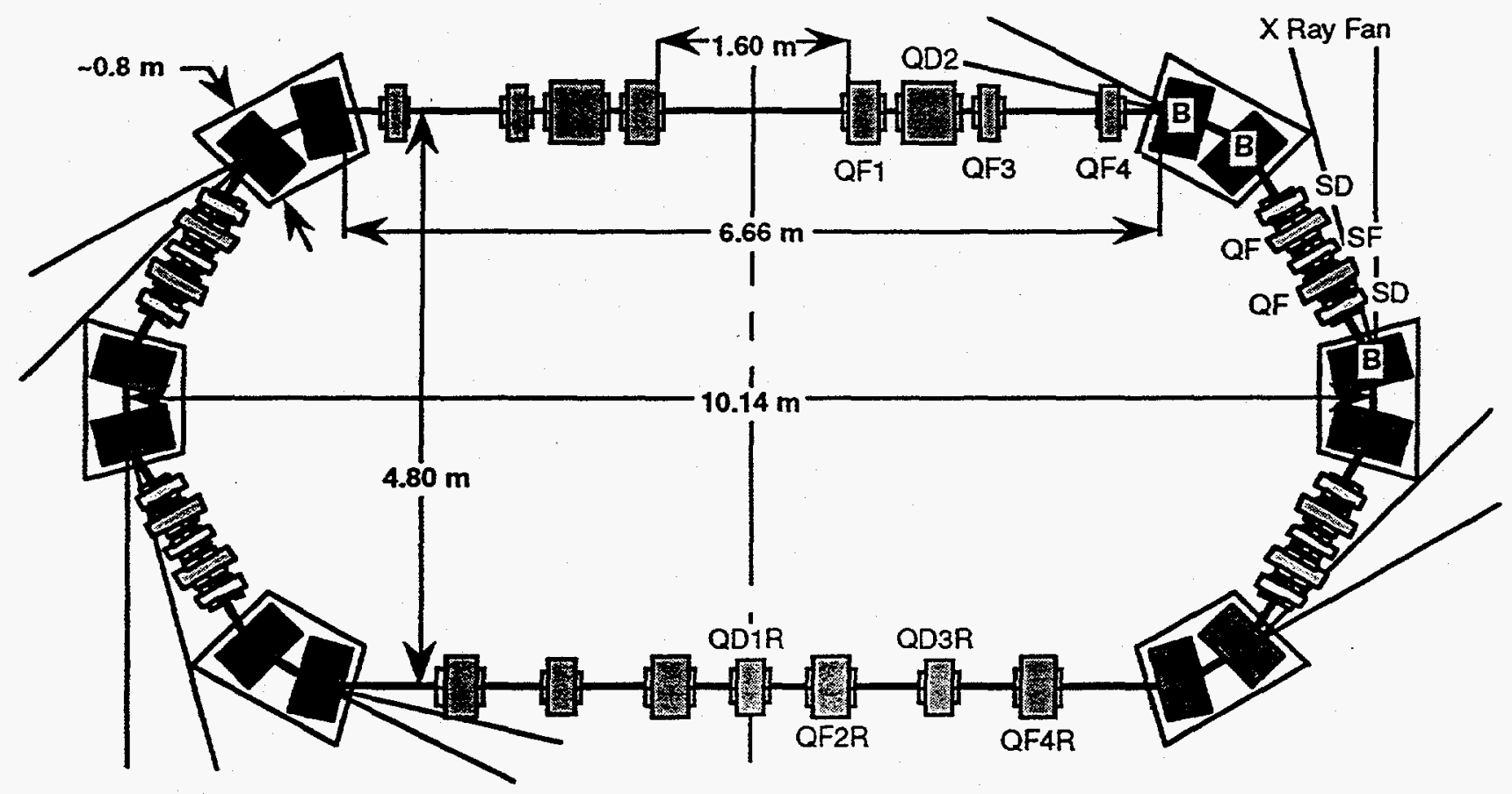

Fig. 1. A Compact Storage Ring with Superconducting Dipoles and Conventional Focusing Elements

Table I Lattice Parameters for the Compact Light Source

Maximum Stored Beam Energy (Gev)

Projected Injection Beam Energy (Gev)

Projected Beam Current (mA)

Circumference (m)

Bend Radius (m)

Dipole Bend Angle (deg)

Integrated Dipole Induction* $(\mathrm{T} \mathrm{m})$

Dipole Central Induction (T)

Dipole Magnetic Length along the Bend (m)

Critical Energy (keV)

Horizontal Natural Emittance* $(\mu \mathrm{m})$

Vertical Coupled Emittance* $(\mu \mathrm{m})$

Vertical Operating Emittance* $(\mu \mathrm{m})$

Horizontal Tune

Vertical Tune

Horizontal Chromaticity

Vertical Chromaticity

Maximum Horizontal Beta Function (m)

Maximum Vertical Beta Function (m)

Maximum Dispersion (m)

Energy Loss per Turn* (MeV/turn)

RF Voltage (MV)

RF Frequency (MHz)

Energy Spread (parts in 1000)

Bunch Length rms* (mm)

Horizontal Damping Time* (ms)

Vertical Damping Time* (ms)

Energy Damping Time* (ms)

Quantum Lifetime (s)

\subsection{0}

0.10

$\sim 20$.

26.0

0.7257

30.

2.6197

6.894

0.380

10.3

2.34

1.17

0.0234

3.17

2.57

$-2.22$

$-5.24$

3.09

6.66

1.29

0.617

2.5

499.

1.52

30.

0.412

0.422

0.213

$2.210^{8}$

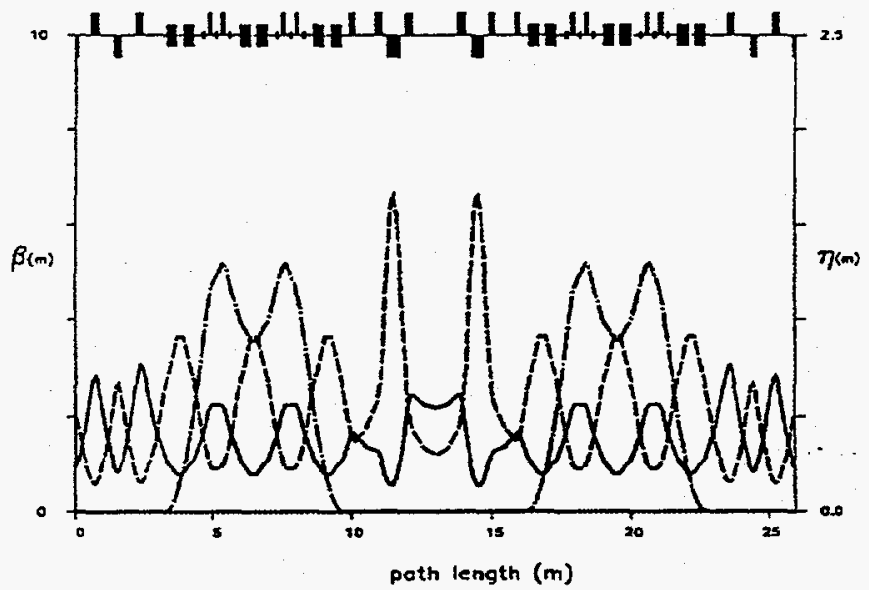

Fig. 2 Orbit Functions of the Compact Storage Ring. The solid, dashed, and dot-dashed curves show $\beta \mathrm{x}$, $\beta \mathrm{y}$, and $\eta$

The comparison of lattice alternatives for the ring, which included engineering and tracking studies, have led to the following conclusions:

1) The split-magnet configuration (six dipoles per arc) is far preferable to the unsplit one.in cost and field quality.

2) The best sextupole configuration is the one that has four SDs per arc.

3) The best vertical cell phase advance is $90^{\circ}$ : this value leads to the shortest feasible arc and the lowest emittance.

4) The best working point is at $v_{x}=3.17, v_{y}=2.57$.

* at the maximum design beam energy 
Particles were tracked for 1,024 turns around the ring. Multipole errors were included in the dipoles consistent with the assumption of dipole field uniformity of 1 part in 10000 over a radial dimension of $28 \mathrm{~mm}$. The dynamic aperture was at least 25 sigma in both planes, considerably larger than the 15 sigma physical aperture of the magnets. This aperture should be more than large enough for beam lifetime.

\section{THE SUPERCONDUCTINGDIPOLE}

Fig. 3 shows a cross-section of the dipole. The design for the dipole is based on a concept proposed by Pavel Vobly at INP Novosibirsk.[2,3]. The dipole shown in Fig. 3 will generate a uniform field over a wide range of central inductions, provided that the current density in the shield coil system is correctly chosen with respect to the current density in the gap and crossover coil system. The shield coil current can be set so that the sextupole and decapole field components are zero, and the 14 pole component is very small. The excitation function can be modified by changing the height and slope of the shield coil.

The dipole has saturated iron in the poles. The shield coils keep the magnetic flux in the pole until it can be returned by an unsaturated iron return yoke. As a result, the field fall off at the end of the dipole is similar to that of conventional low field copper iron dipoles. Since the compact ring dipoles are only $376 \mathrm{~mm}$ long, the fall-off of the magnetic field at the end is important.

If one bases the coil design shown in Figure 3 on superconducting cable made from SSC inner conductor, the dipole will operate at about $87 \%$ of its critical current along the load line (based on a conductor packing fraction of 0.7 and an operating temperature of $4.4 \mathrm{~K}$ ). The SSC conductor can be cabled into conductor of almost any desired current for this application.

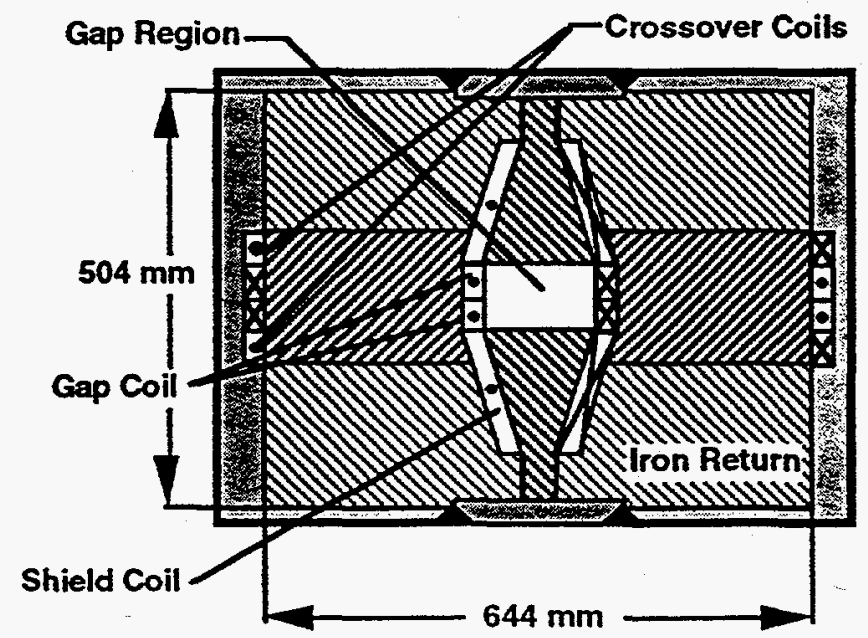

Fig. 3 Cross-section of a 6.9 T Vobly Type Dipole

\section{CONCLUSION}

A $1.5 \mathrm{GeV}$ compact synchrotron with superconducting bending magnets and conventional focusing elements can be made with a circumference of 26 meters. At $1.2 \mathrm{GeV}$, the proposed storage ring should perform even better. The ring appears to be suitable for industrial production of $X$ rays at energies up to $35 \mathrm{keV}$. In this energy. range there are a number of interesting potential uses for such a ring, which, it is expected, can be built for less than $\mathbf{1 2}$ million dollars.

The key to building a compact electron storage ring in the 1.2 to $1.5 \mathrm{GeV}$ energy range is the development of a short superconducting dipole with field characteristics similar to that of conventional room temperature iron dominated dipoles. It appears that a Vobly type dipole is an attractive design for a compact $\mathrm{X}$ ray source.

\section{REFERENCES}

[1] D. B. Cline A. A. Garren, M. A. Green, et al, "A Compact Hard X Ray Source for Medical Imaging and Biomolecular Studies," to be published in NIM (1995), UCLA-CAA0112

[2] G. N. Kulipanov, N. A. Mezentsev, L. G. Morgonov, et al, "Development of a Superconducting Compact Storage Rings for Technical Purposes in the USSR," Rev. Sci. Instrum. 63 (1), p731 (1992)

[3] P. D. Vobly, private communication concerning compact synchrotron dipole magnet designs (1992) 